\title{
The role of a best practice alert in the electronic medical record in reducing repetitive lab tests
}

This article was published in the following Dove Press journal:

ClinicoEconomics and Outcomes Research

\author{
Harini Bejjanki' \\ Lazarus K Mramba ${ }^{2}$ \\ Stacy G Beal ${ }^{3}$ \\ Nila Radhakrishnan' \\ Rohit Bishnoi' \\ Chintan Shah' \\ Nikhil Agrawal ${ }^{4}$ \\ Neil Harris ${ }^{3}$ \\ Robert Leverence \\ Kenneth Rand ${ }^{3}$ \\ 'Division of Hospital Medicine, \\ Department of Medicine, University \\ of Florida, Gainesville, FL, USA; \\ ${ }^{2}$ Statistics, Department of Internal \\ Medicine, University of Florida, \\ Gainesville, FL, USA; ${ }^{3}$ Department of \\ Pathology, Immunology, \& Laboratory \\ Medicine, College of Medicine, \\ University of Florida, Gainesville, FL, \\ USA; ${ }^{4}$ Department of Nephrology, \\ Beth Israel Deaconess Medical Center, \\ Harvard Medical School, Boston, MA, \\ USA
}

Correspondence: Harini Bejjanki Division of Hospital Medicine, Department of Medicine, University of Florida, 1600 SW Archer Road M509, Gainesville, FL 32610, USA

$\mathrm{Tel}+\mathrm{I} 7349858467$

Email dr.harinipal@gmail.com
Background: The recommendations of the American Board of Internal Medicine Foundation's "Choosing Wisely ${ }^{\mathbb{R}}$ " initiative recognize the importance of improving the appropriateness of testing behavior and reducing the number of duplicate laboratory tests.

Objective: To assess the effectiveness of an electronic medical record Best Practice Alert (BPA or "pop up") intervention aimed at reducing duplicate laboratory tests and hospital costs.

Design: Comparison of the number of duplicated laboratory tests performed on inpatients before and after the intervention.

Setting: University of Florida Health Shands Hospital, Gainesville, FL, USA, during 2014-2017. Intervention: The electronic medical record intervention was a BPA pop-up alert that informed the ordering physician if a recent identical order already existed along with the "ordering time", "collecting time", "resulting time", and the result itself.

Main outcome measures: Percentage change in the number of inpatient duplicate orders of selected clinical biochemistry tests and cost savings from reduction of the duplicates. Student's $t$-test and beta-binomial models were used to analyze the data.

Results: Results from the beta-binomial model indicated that the intervention reduced the overall duplicates by $18 \%(\mathrm{OR}=0.82$, standard error $=0.016, P$-value $<0.000)$. Percent reductions in 9 of the 17 tests were statistically significant: serum hemoglobin A1C level, vitamin B12, serum erythrocyte sedimentation rate, serum folate, serum iron, lipid panel, respiratory viral panel, serum thyroid stimulating hormone level, and Vitamin D. Additionally, important cost savings were realized from the reduction of duplicates for each lab test (with the exception of CRP) with an estimated overall savings of $\$ 72,543$ over 17 months in the post-intervention period.

Conclusions: The present study included all hospital inpatients and covered 17 clinical laboratory tests. This rather simple and low-cost intervention resulted in significant reductions in percentage duplicates of several tests and resulted in cost savings. The study also highlights the role of hospitalists in quality improvement.

Keywords: laboratory, testing, health care, costs

\section{Introduction}

According to the data published by Center for Medicare and Medicaid services, National Healthcare expenditure grew $5.8 \%$ to $\$ 3.2$ trillion in 2015 , or $\$ 9,990$ per person, and accounted for $17.8 \%$ of Gross Domestic Product. National health spending is projected to grow at an average rate of 5.6\% per year for 2016-2025, and 4.7 $\%$ per year on a per capita basis. ${ }^{1}$ Despite the enormous spending, the United States still struggles with less than ideal outcomes when compared with other industrialized nations. Reducing costs is a major effort of governments and policymakers. ${ }^{2}$ One 
component of these costs is the wide range and overwhelming number of diagnostic tests. ${ }^{3}$ Laboratory testing of hospitalized patients can be repetitive when multiple providers order tests for the same patient, contributing needlessly to the total health care costs. ${ }^{4-6}$

At the University of Florida - Shands Hospital, we have a relatively higher number of laboratory tests/patient ordered compared with other comparable Vizient (United Healthcare). Our Direct Cost Index (O/E) for the Division of Hospital Medicine is 1.10 where median Direct Cost Index for Vizient hospitals is 0.91 .

Over ordering, under ordering, and misordering of tests are all common, and specific practices vary tremendously from community to community. We do not have local data to show exactly how much of our direct cost is due to lab testing. Redundant and repetitive testing not only adds burden to the health care system but can also lead to increased cost, unnecessary phlebotomy, decreased patient satisfaction, and iatrogenic anemia. ${ }^{7}$ It has been shown that excessive phlebotomy causes patient discomfort and can result in hospitalacquired anemia, which is associated with additional testing, prolonged hospitalizations, unnecessary transfusions, and increased mortality for patients with cardiopulmonary diseases. ${ }^{7-10}$ Laboratory services may make up 5\% of a hospital's budget but leverage $60 \%-70 \%$ of all critical decision-making such as admittance, discharge, and medication. ${ }^{11}$

The Society of Hospital Medicine has listed avoidance of repetitive lab testing as one of their Choosing Wisely ${ }^{\circledR}$ recommendations. ${ }^{14}$ The critical care societies collaborative, which includes the American college of Chest Physicians and American Thoracic Society, has listed avoidance of every day diagnostic tests as their top recommendation. ${ }^{15}$ Studies with interventions including educational campaigns, provider audit and feedback, and changes to ordering options in the electronic medical record (EMR) have shown some success in this area. ${ }^{16,17}$

Some studies have shown that EMR-based strategy of limiting the ability of clinicians to order repetitive laboratory tests leads to a consistent and sustainable reduction in blood draws. The Veterans Affairs System implemented an electronic laboratory utilization management system (Laboratory Expert System [LES]) to provide safe and effective reductions in unnecessary clinical laboratory testing. Since implementing the LES, total test volume has decreased by a mean of $11.18 \%$ per year compared with pre-LES test volume. ${ }^{18}$ However, this study was implemented for three highvolume tests only. The LES offered a significant roadblock to repetitive ordering: ordering clinicians were required to contact the laboratory to have a frequency filter lifted for a given patient. In comparison, our study was implemented on 17 tests and allowed physicians to order the test even after the Best Practice Alert (BPA) alerted the ordering physician.

The objectives of this study were as follows 1 ) to examine the EMR at our institution and determine if initiation of computerized alerts, with evidence-based, test-specific time intervals determined by a multidisciplinary group of physicians including hospitalists and pathologists would reduce duplicate laboratory testing and 2) to determine if overall reduction in laboratory testing can reduce hospital costs.

Our study utilized a multidisciplinary approach with resultant decrease in lab redundancy and cost savings to the hospital.

\section{Methods}

Clinical setting: The site of this study was University of Florida (UF) Health Shands Hospital, a large academic medical center affiliated with UF. UF Health Shands Hospital is a private, not-for-profit tertiary center with 915 beds, of which 232 are intensive care unit (ICU) beds and 127 are intermediate medical care (IMC) beds. The remaining are general beds. This information is obtained from the budget and planning department. The hospitalists cover about $20 \%$ of the patients and there are no residents, fellows, Physician Assistants (PAs), or Nurse Practitioners (NPs) on this service. Most other services in the hospital have residents, fellows, and extenders.

The authors followed the Specific, Measure, Action, Realistic, Timely aim methodology for the quality improvement (QI) work as outlined below.

Specific: We had a specific aim from the inception of the study to decrease the percentage of repetitive labs and follow the results about 1.5 years after the intervention until May 2017.

Measure: Our aim was to decrease the percentage of duplicates by $>50 \%$. We have reduced the percentage of duplicates but did not reach the aim.

Action: The authors of the study worked toward the aim. Our action included creation of the BPA alert and implemented it as a solution.

Realistic: We believed in the possibility of acting toward the aim and successfully achieving it with due assistance from the Information Technology analysts in creating the alert and we had access to specialists to make decisions regarding cutoff windows for each test. 
Timely: The goal had a target date of implementing on a specific date in September 2015 and following the results until a specific period of time.

In September 2014, hospitalists and pathologists formed the "Reducing Lab Costs Team". The team consisted of physicians but ad hoc guests (statisticians, quality officers, and information technology staff) were invited as needed.

First, we identified 17 tests (see Table 1 for test names with definitions), which we believed were frequently ordered as duplicates. We considered a test to be a duplicate if a second test performed within a specified time period would not change patient management. Then, based on test characteristics, published best practice guidelines, and expert opinion from within our institution, we determined the time period for which each individual test would be considered a duplicate. When needed for specific tests, physicians from relevant subspecialties were consulted to establish appropriate time periods.

Subsequently, we collaborated with Information Technology to develop an electronic Best Practice Alert ("pop up") to display in our electronic health record (Epic Systems Corporation, Verona, WI, USA). At the time of order entry in the inpatient setting, the Best Practice Advisory (BPA) would fire if the order was considered a duplicate. The alert was created to display "ordering time", "collecting time", "resulting

Table I Seventeen test names and window periods

\begin{tabular}{|l|l|}
\hline Test & Window period \\
\hline CBC w diff & I day=24 hours \\
BNP & I day=24 hours \\
BI2 & I month=730 hours \\
Ferritin & I month=730 hours \\
Iron & I month=730 hours \\
Folate & I month=730 hours \\
Lipid panel & I month=730 hours \\
PSA & I month=730 hours \\
CRPHS & I week=168 hours \\
ESR & I week=168 hours \\
ANA & I week=168 hours \\
Free T4 & 2 weeks=336 hours \\
Ammonia & 2 weeks=336 hours \\
TSH & 2 weeks=336 hours \\
RVP & 3 days=72 hours \\
HBAIC & 3 months=219I hours \\
Vitamin D & 3 weeks=504 hours \\
\hline
\end{tabular}

Notes: ammonia, serum ammonia level; ANA, serum antinuclear antibody; BI2, serum Vitamin BI2; BNP, serum B natriuretic peptide; CBC w diff, complete blood count with differential; $C R P H S$, serum high sensitivity $C$ reactive protein; $E S R$, serum erythrocyte sedimentation rate; ferritin, serum ferritin level; folate, serum folate level; free T4, serum free T4 level; HBAIC, serum hemoglobin AIC level; iron, serum iron saturation level; PSA, serum prostate specific antigen; RVP, respiratory viral panel - nasopharynx swab; TSH, serum thyroid-stimulating hormone level. time", and the recent result. The user had the capability to elect to continue with the duplicate order if required.

The alert went into practice for the 17 laboratory tests on September 29, 2015, for all inpatient units. The Emergency Department was excluded. The team continued to meet monthly after implementation to monitor test volumes, cost savings, and other test utilization patterns to detect and identify workaround efforts (ie, placing an order via order sets), establish new test ordering rules, or edit existing rules. There were no other specific hospital-wide or department initiatives to reduce unnecessary laboratory testing for the 17 analytes in our study during the time of our study. Besides the alert, there were no other interventions such as providers' education or feedback.

After implementation of the BPA, the hospital's data analysts retrieved the data using the structured query language via Epic Clarity database (current version is Epic 2017). An automated data retrieval process was scheduled to run on the fifth day of the month, every 3 months (ie, quarterly). The alert was spread hospital wide to ICUs, IMC units, and general beds. The data were retrieved for all inpatient units in the hospital including all ICUs, IMCs, and general beds. Unit-based data were not obtained for this study, but we plan to obtain that data in future. Since only inpatients are included, patient complexity was at least moderate to high.

\section{Statistical analysis}

Descriptive analysis was performed using two-sample $t$-test to compare differences in mean proportions of duplicates pre and post intervention. (Results are provided in Table 2.)

Significance was set at 5\% level using a two-tailed test. Beta-binomial regression models were used to analyze the percentage of duplicates reduction between the pre- and post-intervention periods (Table 3).

Duplicate expenses were computed by multiplying the unit cost for a given lab test by the number of duplicates ordered for that specific test. Savings were defined as the difference between the before and after intervention duplicate expenses.

Analysis was done using Stata Statistical Software v. $15 .^{12}$

\section{Ethics approval}

A statement from the director of the Institutional review board of the University of Florida (IRB) waiving the requirement for an IRB based on the QI nature of the project was obtained on October 6, 2014, IRB approval was later obtained in 2017 for a retrospective chart review study. Data were analyzed for 34 months, 17 months pre intervention (May 2014 to 
Table 2 Mean differences in percentage duplicates before and after intervention

\begin{tabular}{|l|l|l|l|l|l|}
\hline & $\begin{array}{l}\text { Mean difference } \\
\text { \% duplicates }\end{array}$ & SE & $\mathbf{9 5 \%} \mathbf{C l}$ & P-value & $\begin{array}{l}\text { \% change = } \\
\text { (diff/before) } \times \mathbf{I 0 0}\end{array}$ \\
\hline Overall & -1.78 & 0.80 & {$[0.21,3.35]$} & 0.03 & $15.4 \%$ \\
HbAIC & -3.32 & 0.54 & {$[2.23,4.4 I]$} & 0.00 & $28.2 \%$ \\
ANA & -0.31 & 0.52 & {$[-0.75,1.36]$} & 0.56 & $27.4 \%$ \\
Ammonia & -3.02 & 2.10 & {$[-1.26,7.3 I]$} & 0.16 & $25 \%$ \\
BI2 & -3.14 & 0.41 & {$[2.30,3.98]$} & 0.00 & $49.6 \%$ \\
CBC w diff & 0.87 & 0.79 & {$[-2.47,0.73]$} & 0.27 & $3.3 \%$ (increase) \\
CRP & 1.92 & 0.67 & {$[-3.27,-0.56]$} & 0.01 & $7.8 \%$ (increase) \\
ESR & -4.71 & 0.82 & {$[3.03,6.38]$} & 0.00 & $28 \%$ \\
Ferritin & -1.71 & 1.03 & {$[-0.39,3.8 I]$} & 0.11 & $14.7 \%$ \\
Folate & -1.69 & 0.43 & {$[0.82,2.55]$} & 0.00 & $40.7 \%$ \\
Free T4 & -1.23 & 0.74 & {$[-0.28,2.74]$} & 0.11 & $9.8 \%$ \\
Iron & -2.33 & 0.55 & {$[1.21,3.45]$} & 0.00 & $33.3 \%$ \\
Lipid & -1.97 & 0.46 & {$[1.03,2.90]$} & 0.00 & $28.6 \%$ \\
BNP & -0.49 & 0.31 & {$[-0.14,1.1 I]$} & 0.12 & $12.5 \%$ \\
PSA & -0.29 & 1.78 & {$[-3.34,3.92]$} & 0.87 & $6.9 \%$ \\
RVP & -2.07 & 0.45 & {$[1.15,2.98]$} & 0.00 & $33.4 \%$ \\
TSH & -3.14 & 0.41 & {$[2.30,3.97]$} & 0.00 & $25.1 \%$ \\
Vitamin D & -3.65 & 0.61 & {$[2.41,4.89]$} & 0.00 & $58.7 \%$ \\
\hline
\end{tabular}

Notes: ammonia, serum ammonia level; ANA, serum antinuclear antibody; BI2, serum Vitamin BI2; BNP, serum B natriuretic peptide; CBC w diff, complete blood count with differential; CRP, serum $C$ reactive protein; ESR, serum erythrocyte sedimentation rate; ferritin, serum ferritin level; Folate, serum folate level; free T4, serum free T4 level; HBAIC, serum hemoglobin AIC level; Iron, serum iron saturation level; PSA, serum prostate specific antigen; RVP, respiratory viral panel - nasopharynx swab; TSH, serum thyroid-stimulating hormone level.

Abbreviations: diff, difference; SE, standard error.

Table 3 Results from beta-binomial models showing the odds of percentage duplicates after intervention compared to before intervention

\begin{tabular}{|l|l|l|l|l|}
\hline $\begin{array}{l}\text { After vs before } \\
\text { intervention }\end{array}$ & OR & Rob SE & $\mathbf{P}>\mathbf{z}$ & $\mathbf{9 5 \%} \mathbf{C l}$ \\
\hline Overall & 0.82 & 0.02 & 0.00 & {$[0.79,0.85]$} \\
HbAIC & 0.69 & 0.04 & 0.00 & {$[0.62,0.78]$} \\
ANA & 0.49 & 0.31 & 0.26 & {$[0.14,1.70]$} \\
Ammonia & 0.88 & 0.09 & 0.22 & {$[0.71,1.08]$} \\
BI2 & 0.49 & 0.04 & 0.00 & {$[0.42,0.57]$} \\
CBC w diff & 1.04 & 0.04 & 0.28 & {$[0.97,1.12]$} \\
CRP & 1.10 & 0.04 & 0.01 & {$[1.02,1.19]$} \\
ESR & 0.68 & 0.05 & 0.00 & {$[0.59,0.79]$} \\
Ferritin & 0.82 & 0.10 & 0.13 & {$[0.64,1.05]$} \\
Folate & 0.59 & 0.08 & 0.00 & {$[0.45,0.77]$} \\
Free T4 & 0.88 & 0.06 & 0.05 & {$[0.77,1.00]$} \\
Iron & 0.65 & 0.04 & 0.00 & {$[0.57,0.74]$} \\
Lipid & 0.70 & 0.06 & 0.00 & {$[0.58,0.83]$} \\
BNP & 0.88 & 0.07 & 0.12 & {$[0.75,1.03]$} \\
PSA & 0.74 & 0.42 & 0.60 & {$[0.24,2.26]$} \\
RVP & 0.65 & 0.06 & 0.00 & {$[0.54,0.78]$} \\
TSH & 0.72 & 0.03 & 0.00 & {$[0.67,0.78]$} \\
Vitamin D & 0.40 & 0.04 & 0.00 & {$[0.33,0.49]$} \\
\hline
\end{tabular}

Notes: ammonia, serum ammonia level; ANA, serum antinuclear antibody; BI2, serum vitamin $B$ I2; $B N P$, serum $B$ natriuretic peptide; $C B C$ w diff, complete blood count with differential; $C R P$, serum $C$ reactive protein; $E S R$, serum erythrocyte sedimentation rate; ferritin, serum ferritin level; folate, serum folate level; free T4, serum free T4 level; HBAIC, serum hemoglobin AIC level; iron, serum iron saturation level; PSA, serum prostate specific antigen; RVP, respiratory viral panel nasopharynx swab; TSH, serum thyroid-stimulating hormone level.

Abbreviations: Rob, robust; SE, standard error.
September 2015) and 17 months post intervention (October 2015 to February 2017).

Patient consent to review the medical records was not required by IRB. IRB waiver was obtained as the retrieval of data required Information Technology Analyst's help using structured query language. The authors of the study were provided de-identified data during the specified time period without need for chart review or access to HIPAA information, covering patient data confidentiality. For example, data were provided in excel format as "test name", "month", "year" "number of tests ordered for each test in a certain month".

\section{Results}

Based on the two-sample $t$-test, the average percentage duplicates ( \pm standard error) before and after intervention were $11.7 \% \pm 0.558,95 \%$ CI $[10.6 \%, 12.8 \%]$, and $9.9 \% \pm 0.576$, $95 \%$ CI $[8.8 \%, 11.0 \%]$, respectively, with a difference of $1.8 \% \pm 0.802,95 \%$ CI $[0.21 \%, 3.4 \%](P$-value $=0.027)$. The overall percentage reduction in duplicates after intervention is estimated to be $(1.8 / 11.7) \times 100 \%=15.4 \%$. Table 2 provides summary statistics for each of the lab test's mean differences before and after intervention using the Student's $t$-test. Mean difference percentage duplicate is defined as mean percentage 
duplicates before intervention - mean percentage duplicates after intervention for that particular test. Percentage difference is mean duplicate percentage before intervention - mean duplicate percentage after intervention/ mean duplicate percentage before intervention $\times 100$.

Results from the beta-binomial model fitted with robust Huber's (sandwich) standard errors indicated that the odds of duplicates after intervention was reduced by $18 \%$ (OR $=0.82,95 \%$ CI $[0.79,0.85]), P$-value $<0.001$; Table 3 ). Figures 1-3 show marginal proportions of duplicates before and after the intervention obtained from the fitted beta-binomial models. Figure 1 is obtained by aggregating all the tests together resulting in an overall percentage of duplicates for all tests combined, whereas Figures 2 and 3 represent the mean proportion of duplicates before and after each lab test.

Based on the beta-binomial model all the specific lab tests shown in Table 3 had their odds of duplicates reduction being statistically significant at 5\% level except for serum antinuclear antibody, ammonia, $\mathrm{CBC}$ with differential, ferritin, serum $B$ natriuretic peptide (BNP), and serum prostate specific antigen, which showed a downward trend while serum free T4 level was at borderline $(P$-value $=0.05)$. CRP showed an increase by $10 \%$ after intervention $(\mathrm{OR}=1.10$. 95\% CI [1.02-1.19], $P$-value $<0.05)$.

The actual hospital laboratory direct test cost was provided by hospital administration. The cost of duplicates pre-intervention was $\$ 8,63,681.95$, and the cost of duplicates after intervention is $\$ 7,91,138.11$. This is calculated by multiplying the cost of each test by the number of duplicates for that test (Table 4).

We found that computerized alert intervention was effective in decreasing the total number of inpatient orders, the

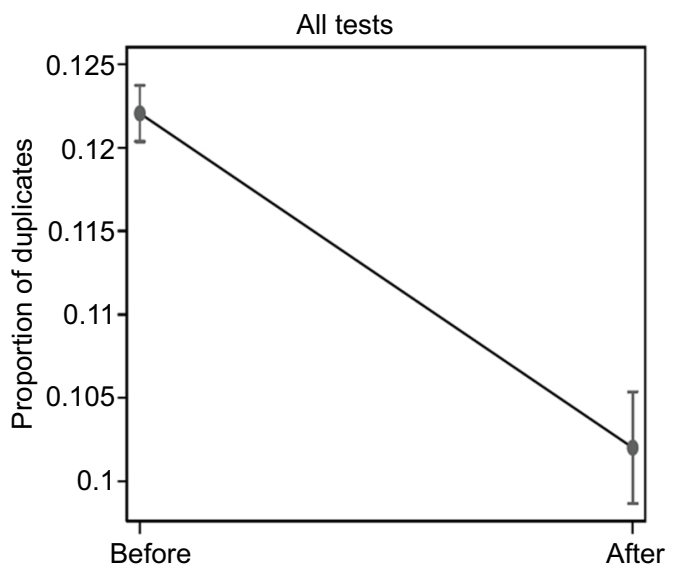

Figure I Overall proportion of duplicates before and after intervention. total number of inpatient duplicate orders, and the percentage of total duplicate orders. The total inpatient orders during the pre-intervention period from May 01, 2014, to September 30, 2015, were 3,95,650 including all 17 tests. The total inpatient orders during the post-intervention period from October 01, 2015 , to February 28, 2017, were 3,66,051. The total number of inpatient duplicate orders before the intervention was 83,951 , and the total number after intervention was 77,997 . The overall percentage reduction in duplicates after intervention was $15.4 \%$, which is statistically significant.

CBC with differential showed a decrease in the total number of inpatient orders (from 2,45,847 to 2,26,751) and the total number of duplicate inpatient orders after implementation (from 63,674 to 60,520). However, there was a percentage increase in the duplicates after intervention $(3.3 \%$; see Table 2). Of note, total inpatient days during the preintervention period are $3,25,734$. Total inpatient days during post-intervention period are 3,72,726, suggesting the number of ordered tests during post-intervention period would have been lower if adjusted for inpatient days.

The cost-effective analysis showed significant cost savings of $\$ 72,543$ for all 17 tests in the post-implementation period. Only CRP showed no savings with respect to expenditure on duplicates test orders.

\section{Discussion}

Modern computer systems allow limits to be set on the periods allowed for repetitive testing. The results of our study are comparable to a study published in 2013 in Netherlands. The study investigated a computerized system for managing potentially overfrequent laboratory testing, calculating the financial savings obtained. Tests were selected for which "spare periods" (periods during which tests are barred) might be set to control repetitive testing. The proportion of tests barred was $0.56 \%$, and the financial savings was $0.33 \%$ of the costs of all testing. Following the introduction of the system, the number of barred tests ultimately decreased, suggesting accommodation by the test requestors. ${ }^{19}$ Our study supports the findings of this study.

Surprisingly, CRP is the only test that showed a statistically significant increase in the percentage duplicates after intervention. We confirmed that the alert was working appropriately and investigated any possible order sets which it was added to but found no way to explain this increase. Some providers recommend monitoring of CRP daily curve (at minimum time intervals of 24 hours), with verification of a trend of reduction or increase, provided additional data to the daily clinical evaluation of the patient. ${ }^{13}$ 

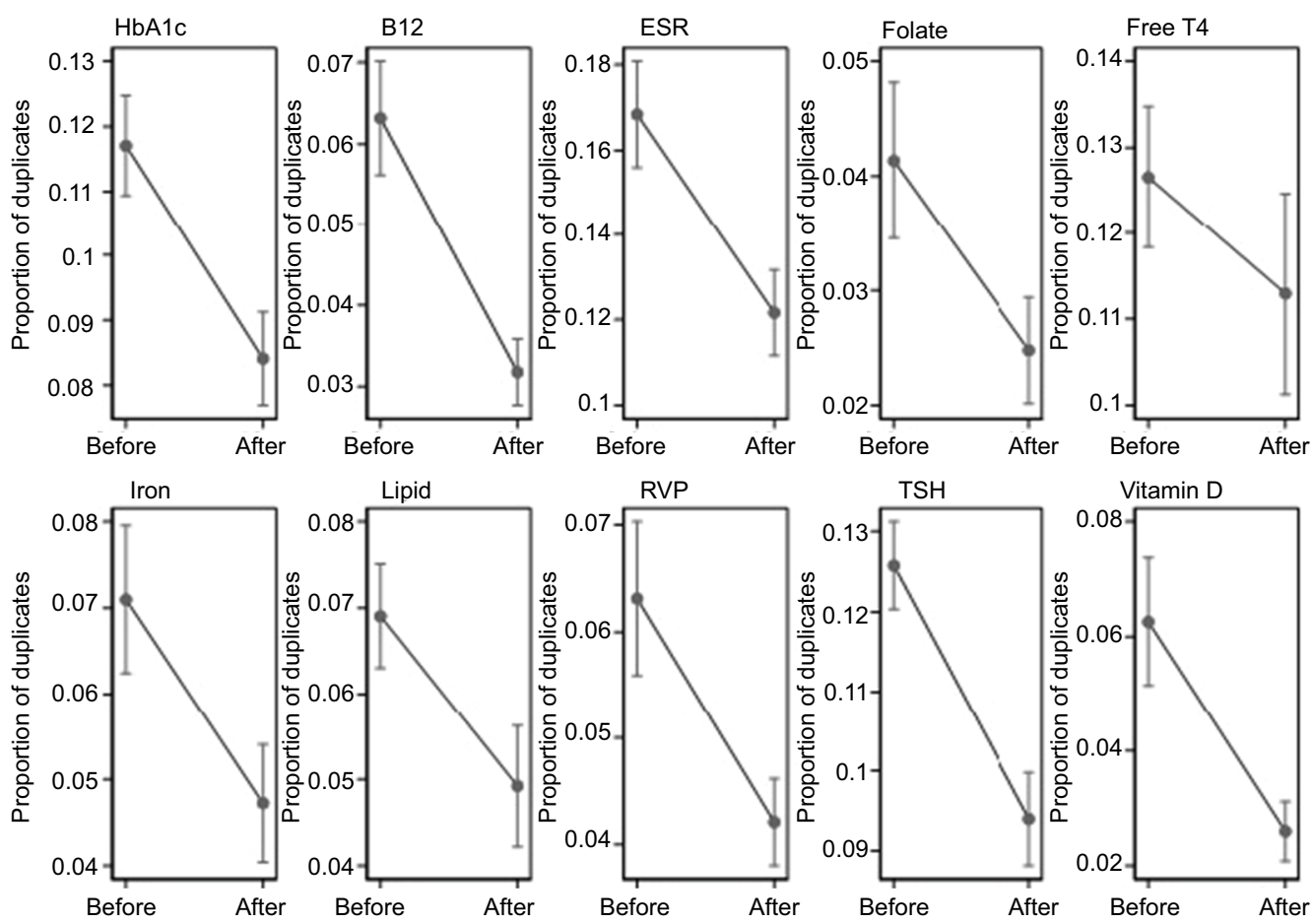

Figure 2 Lab test's specific proportion of duplicates before and after intervention (tests with statistically significant decrease in the duplicates after intervention). Notes: BI2, serum vitamin BI2; ESR, serum erythrocyte sedimentation rate; folate, serum folate level; free T4, serum free T4 level; HbAIc, serum hemoglobin AIC level; iron, serum iron saturation level; RVP, respiratory viral panel - nasopharynx swab; TSH, serum thyroid-stimulating hormone level.

We plan to do a chart review and provide education and feedback to providers, but we expect this will take time and resources beyond the scope of the present study.

A pre-post study in which the ability to order repetitive laboratory tests was removed from the admission order set resulted in a nearly $20 \%$ decrease $(P<0.001)$ in laboratory tests ordered per patient-day. ${ }^{20}$ In our EMR system, we have several order sets including admission order set and sepsis order set, among others. We implemented the BPA in such a way that it alerts the ordering physician even if the duplicate lab order is ordered from the order sets.

Repeat and/or serial BNP testing is inappropriate for guiding the management of heart failure and may be clinically misleading. The clinical decision support intervention implemented by Lehigh Valley health system consists of an expert rule that searches the system for a BNP lab value on the patient. If there is a value and the value is within the current hospital stay, an advisory is displayed to the ordering clinician. Multiple regression analysis results suggest that the intervention reduced BNP orders by $21 \%$ relative to the mean. ${ }^{21}$ Our study showed that the odds of duplicates after intervention for BNP was reduced by $12 \%$, supporting the findings.
A general inpatient ward identified five tests commonly ordered. In order to minimize repetitive tests, a multidisciplinary committee of that hospital developed the electronic order function to limit a particular laboratory test request to occur once within one 24-hour window. Comparison of fiscal year volume data from before (2002-2003) and after (2003-2004) implementation revealed 72,639 (12.0\%) fewer inpatient tests, of which 41,765 (57.5\%) were related directly to decreases in the five tests frequently ordered on a recurring basis. ${ }^{22}$ Our study showed 29,599 fewer tests in the 17 frequently ordered tests in the post-implementation period. When corrected to adjusted inpatient days, the number of ordered tests during the post-intervention period would have been even lower.

Our study has several limitations. The study was conducted in a single tertiary care organization, limiting the generalizability of our study to other facilities and settings. The study did not consider the charge of duplicate testing to patients or secondary payers. We did not analyze the reasons behind why physicians ordered tests despite the intervention alert. While both length of stay and readmission rates improved during the study period, there were multiple other projects dedicated to improving these measures. Therefore, 

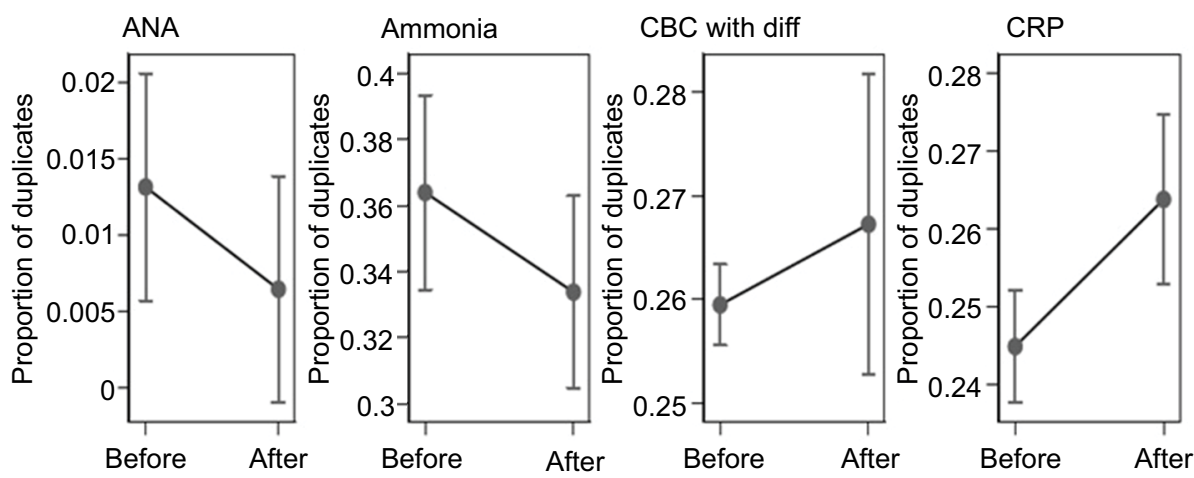

Ferritin

NT ProBNP

PSA
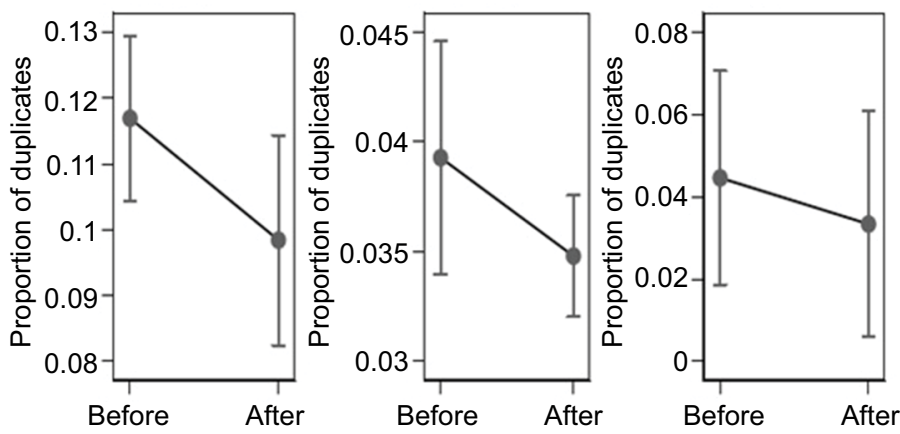

Figure 3 Lab tests' specific proportion of duplicates before and after intervention.

Notes: ammonia, serum ammonia level; ANA, serum antinuclear antibody; $C B C$ w diff, complete blood count with differential; CRP, serum C reactive protein; ferritin, serum ferritin level; PSA, serum prostate specific antigen.

Table 4 Cost of 17 tests and cost savings after intervention in US dollars (\$)

\begin{tabular}{|c|c|c|c|c|}
\hline Test & Cost of test & Cost (before) & Cost (after) & $\begin{array}{l}\text { Difference } \\
\text { (before - after) }\end{array}$ \\
\hline $\mathrm{HbAIC}$ & 14.53 & $20,196.7$ & $12,844.52$ & $7,352.18$ \\
\hline Ammonia & 11.96 & $22,197.76$ & $18,968.56$ & $3,229.2$ \\
\hline ANA & 23.19 & $30 \mathrm{I} .47$ & 92.76 & 208.71 \\
\hline $\mathrm{B} \mid 2$ & 14.84 & $6,603.8$ & $2,953.16$ & $3,650.64$ \\
\hline CBC w diff & 8.52 & $5,42,502.48$ & $5,15,630.4$ & $26,872.08$ \\
\hline CRP & 13.19 & $\mathrm{I}, 05,295.77$ & $\mathrm{I}, 20,424.7$ & $-15,128.9$ \\
\hline ESR & 7.05 & $1 \mathrm{I}, 886.3$ & $6,789.15$ & $5,097.15$ \\
\hline Ferritin & 12.97 & $7,639.33$ & $6,796.28$ & 843.05 \\
\hline Folate & 13.73 & $3,240.28$ & $\mathrm{I}, 798.63$ & $\mathrm{I}, 44 \mathrm{I} .65$ \\
\hline Free T4 & 12.31 & $15,597.6$ & $11,081.28$ & $4,516.32$ \\
\hline Iron & 6.57 & $2,319.21$ & $\mathrm{I}, 583.37$ & 735.84 \\
\hline Lipid & 11.64 & $8,904.6$ & $4,574.52$ & $4,330.08$ \\
\hline ProBNP & 47.75 & $31,276.25$ & 27,886 & $3,390.25$ \\
\hline PSA & 20.03 & 360.54 & 260.39 & 100.15 \\
\hline RVP & 106.97 & $46,531.95$ & $33,695.55$ & $12,836.4$ \\
\hline TSH & 16.39 & $38,827.91$ & $24,519.44$ & $14,308.47$ \\
\hline Vitamin D & 20 & 2,880 & $\mathrm{I}, 240$ & $\mathrm{I}, 640$ \\
\hline Grand total & 21.4 & $8,63,681.95$ & $7,91,138.7$ & $72,543.24$ \\
\hline
\end{tabular}

Notes: ammonia, serum ammonia level; ANA, serum antinuclear antibody; BI2, serum vitamin BI2; BNP, serum B natriuretic peptide; CBC w diff, complete blood count with differential; CRP, serum C reactive protein; ESR, serum erythrocyte sedimentation rate; ferritin, serum ferritin level; folate, serum folate level; free T4, serum free T4 level; HBAIC, serum hemoglobin AIC level; Iron, serum iron saturation level; PSA, serum prostate specific antigen; RVP, respiratory viral panel - nasopharynx swab; TSH, serum thyroid-stimulating hormone level.

we are unable to state the impact of our project, if any, on length of stay or readmission rate. There is no way to measure delayed diagnosis or patient/family satisfaction in a de-identified population.

\section{Conclusions}

Overall, our study showed that the EMR-based strategy of limiting the ability of clinicians to order repeating laboratory tests leads to a consistent and sustainable reduction in 
blood draws similar to few other studies already discussed. Our study is unique in that we implemented the intervention across 17 tests and collected data ranging over a period of 3 years allowing room for random variation and statistically more reliable results. Our study determined that implementation of computerized alerts, with evidence-based, timespecific intervals, reduced redundant laboratory testing, and resulted in cost savings for the hospital. This approach could potentially be expanded to other tests and may be applicable in other institutions.

Future endeavors could include investigating the reasons for the overridden tests, obtaining and analyzing data based on each unit (ICU, IMC, general beds), obtaining and analyzing data based on the type of ordering providers (Attendings, Residents/Fellows, NPs/PAs), and providing education and feedback to ordering providers. Another significant step is showing the changes in duplicative lab tests in tests that were not included in the study. The retrieval of additional data including unit-based data requires assistance from IT staff and funding.

\section{Disclosure}

The authors report no conflicts of interest in this work.

\section{References}

1. NHE Fact Sheet, 2016. National Health Expenditure Projections 2016-2025. Available from: https://www.cms.gov/Research-Statistics-Data-and-Systems/Statistics-Trends-and-Reports/NationalHealthExpendData/Downloads/proj2016.pdf. Accessed September 23, 2018.

2. DesRoches CM, Campbell EG, Vogeli C, et al. Electronic health records' limited successes suggest more targeted uses. Health Aff (Millwood). 2010;29(4):639-646.

3. Jackson BR. Managing laboratory test use: principles and tools. Clin Lab Med. 2007;27(4):733-748.

4. Bates DW, Boyle DL, Rittenberg E, et al. What proportion of common diagnostic tests appear redundant? Am J Med. 1998;104(4): 361-368.

5. Kwok J, Jones B. Unnecessary repeat requesting of tests: an audit in a government hospital immunology laboratory. $J$ Clin Pathol. 2005;58(5):457-462.
6. van Walraven C, Naylor CD. Do we know what inappropriate laboratory utilization is? A systematic review of laboratory clinical audits. JAMA. 1998;280(6):550-558.

7. Languasco A, Cazap N, Marciano S, et al. Hemoglobin concentration variations over time in general medical inpatients. $J$ Hosp Med. 2010;5(5):283-288.

8. Koch CG, Li L, Sun Z, et al. Hospital-acquired anemia: prevalence, outcomes, and healthcare implications. J Hosp Med. 2013;8(9):506-512.

9. Salisbury AC, Reid KJ, Alexander KP, et al. Diagnostic blood loss from phlebotomy and hospital-acquired anemia during acute myocardial infarction. Arch Intern Med. 2011;171(18):1646-1653.

10. Thavendiranathan P, Bagai A, Ebidia A, Detsky AS, Choudhry NK. Do blood tests cause anemia in hospitalized patients? The effect of diagnostic phlebotomy on hemoglobin and hematocrit levels. J Gen Intern Med. 2005;20(6):520-524.

11. Forsman RW. Why is the laboratory an afterthought for managed care organizations? Clin Chem. 1996;42(5):813-816.

12. StataCorp. Stata Statistical Software: Release 15.College Station, TX: StataCorp LLC;2017.

13. Aguiar FJB, Ferreira-Júnior M, Sales MM, et al. Proteína C reativa: aplicações clínicas e propostas para utilização racional. Revista da Associação Médica Brasileira. 2013;59(1):85-92.

14. Society of Hospital Medicine - Adult Hospital Medicine; 2013. Five Things Physicians and Patients Should Question. Available from: http:// www.choosingwisely.org/wp-content/uploads/2015/02/SHM-AdultChoosing-Wisely-List.pdf. Accessed September 23, 2018.

15. Critical Care Societies Collaborative-Critical Care; 2014. Five Things Physicians and Patients Should Question. Available from: http://www. choosingwisely.org/societies/critical-care-societies-collaborativecritical-care/2014. Accessed September 23, 2018.

16. Silvestri MT, Xu X, Long T, et al. Impact of cost display on ordering patterns for hospital laboratory and imaging services. J Gen Intern Med. 2018;33(8):1268-1275.

17. Minerowicz C, Abel N, Hunter K, Behling KC, Cerceo E, Bierl C. Impact of weekly feedback on test ordering patterns. Am J Manag Care. 2015;21(11):763-768.

18. Konger RL, Ndekwe P, Jones G, et al. Reduction in unnecessary clinical laboratory testing through utilization management at a US Government Veterans Affairs Hospital. Am J Clin Pathol. 2016;145(3):355-364.

19. Janssens PM, Wasser G. Managing laboratory test ordering through test frequency filtering. Clin Chem Lab Med. 2013;51(6):1207-1215.

20. Sadowski BW, Lane AB, Wood SM, Robinson SL, Kim CH. High-value, cost-conscious care: iterative systems-based interventions to reduce unnecessary laboratory testing. Am J Med. 2017;130(9):1112.e1-1112.e7.

21. Levick DL, Stern G, Meyerhoefer CD, Levick A, Pucklavage D. Reducing unnecessary testing in a CPOE system through implementation of a targeted CDS intervention. BMC Med Inform Decis Mak. 2013;13:43.

22. May TA, Clancy M, Critchfield J, et al. Reducing unnecessary inpatient laboratory testing in a teaching hospital. Am J Clin Pathol. 2006;126(2):200-206.
ClinicoEconomics and Outcomes Research

\section{Publish your work in this journal}

ClinicoEconomics and Outcomes Research is an international, peerreviewed open-access journal focusing on health technology assessment, pharmacoeconomics and outcomes research in the areas of diagnosis, medical devices, and clinical, surgical and pharmacological intervention. The economic impact of health policy and health systems

\section{Dovepress}

organization also constitute important areas of coverage. The manuscript management system is completely online and includes a very quick and fair peer-review system, which is all easy to use. Visit http://www.dovepress.com/testimonials.php to read real quotes from published authors. 